\title{
Diagnosis and management of headaches in young people and adults: summary of NICE guidance
}

Devina Halsall was incorrectly omitted from the list of the Guideline Development Group's members in the acknowledgment section of this Practice article (BMJ 2012;345:e5765, doi:10.1136/bmj.e5765).
Cite this as: BMJ 2012;345:e6598

๑ BMJ Publishing Group Ltd 2012 